\title{
Feasibility study on the use of fast camera and recording in time-domain to characterize ESD
}

\author{
Szymon Ptak ${ }^{1, *}$, Albert Smalcerz ${ }^{1}$, Piotr Ostrowski ${ }^{1}$ \\ ${ }^{1}$ Central Institute for Labour Protection - National Research Institute, 16 Czerniakowska St., 00-701 \\ Warsaw, Poland
}

\begin{abstract}
Fire and explosion protection in industrial conditions requires multidimensional approach. Usually the risk of hazardous zone creation is unavoidable, if the combustible material is processed. Therefore controlling of potential ignition sources is introduced. One of most popular sources of ignition is electrostatic discharge. Depending on the type of the discharge, as well as on exact discharge conditions, energy released might reach hundreds or even thousands of $\mathrm{mJ}$, being able to ignite most of gaseous or dust-air hazardous mixtures. A dedicated methodology was created to record the discharge with fast camera with maximum speed of $1 \mathrm{M}$ fps and with the oscilloscope up to $25 \mathrm{GS} / \mathrm{s}$. Dedicated test stand allows to obtain high voltage to create the conditions for electrostatic discharge. The aim of presented research was to analyze the course of electrostatic spark discharge in laboratory conditions and to place the outcomes in the context of explosion safety in the industrial conditions. The course of electrostatic discharge is dependent on various conditions: the polarity, distance between the electrodes, shape of electrodes, grounding conditions, etc. Understanding of the phenomenon is crucial from the point of view of explosion safety.
\end{abstract}

\section{Introduction}

In every industrial process the safety of people, equipment and environment must be ensured. Despite the safety of the process itself, the risk of fire and/or explosion should be always taken into consideration. It might be assumed, that in every industry, in which the combustible material is being processed the explosion hazard should be analyzed and appropriate safety measures are to be taken.

EN 1127 standard describes as much as 13 potential sources of ignition of an explosive atmosphere. Technological process might be therefore analyzed in the scope of the exhaustive threat catalog provided by the above mentioned standard. Among the most typical potential ignition sources following should be mentioned:

- Mechanical spark - considerably small fraction of the material, that was ripped off from the physical body, characterized by significant temperature caused by the friction; typical for electric rotating machinery, e.g. if the coaxiality was lost.

- Surface temperature - every mixture of combustible material with air, as well as

\footnotetext{
*Corresponding author: sptak@sgsp.edu.pl
} 
dust deposit, might be ignited, if the surface temperature reached specific value for particular material.

- $\quad$ Flame - if present, always constitutes an effective source of ignition.

- Static electricity - electric charge might be present on both conductive (not grounded) or non-conductive material. In specific conditions, the charge might be released, during the electrostatic discharge. Significant energy will be therefore transferred to the ambient atmosphere.

Among remaining sources of ignition ones will find the following: electrical apparatus, stray electrical currents (and cathodic corrosion protection), lightning, electromagnetic waves, ionizing radiation, ultrasound and adiabatic compression and shock waves.

In the ATEX directive, the concept of integrated explosion safety was introduced. The main principle is to avoid the formation of an explosive atmospheres, which not always might be ensured in typical industrial processes. On the other hand, uncontrolled release of the combustible substance to the vicinity of particular installation/apparatus might be an effect of a malfunction or an accident. Therefore the second safety barrier should be focused on controlling of potential ignition sources described before.

The third principle orders to predict the effects of the explosion and to implement suitable safety measures. The principles should be considered consecutively to ensure appropriate safety level.

The aim of the article is to present the results of the research on the course of the spark discharge in the context of explosion safety.

\section{Electrostatic discharge}

\subsection{Types of ESD}

There are various types of the electrostatic discharges described in the literature. Basically, if two physical objects have different electrical potentials, they will struggle to reach mutual balance, if favorable conditions are created. Therefore the protection of conductive materials against electrostatic charge accumulation is being realized by appropriate grounding techniques. The situation is not that easy in case of insulating materials. They might be charged by friction and/or induction. Below, most important types of electrostatic discharges (ESD) were briefly described [1-2].

- Spark discharge - usually will discharge the whole volume of the physical object. A plasma channel is being created between conductive electrodes, if the applied voltage exceeds the value of dielectric strength of the air. The discharge time is short, typically in the magnitude of dozens of nanoseconds, energy released significant (in practice, in the range of $10^{-2}$ to $10^{4} \mathrm{~mJ}$ ), also the blast effect was observed in case of highly energetic spark discharges.

- Brush discharge - if the dielectric is charged, as the grounded object approaches, brush discharge might occur. It is typical for spherical (grounded) objects of certain radius. As the distance between the electrode and the dielectric body must be low, the electric field will reach significant values. Therefore the energy released might be high, being able to ignite most of the explosive atmospheres.

- Propagating brush discharge - in some optimal conditions, propagating brush discharge might be observed on the surface of a dielectric foil. If the voltage exceeding the breakdown voltage of the dielectric object? was reached (caused by the charge on the dielectric surface), a spontaneous phenomenon of the propagating brush discharge is observed. It is continued by progressive discharges, so the energy released might reach the values of single joules. It constitutes a hazard not only from the perspective of potential 
explosion, but also might interfere with the electronic devices in the vicinity, as well as might cause severe physiological shocks to the personnel working nearby.

- Corona discharge - in case of needle-shaped electrodes (i.e. of low curvature radius) and the corona discharge might be observed. The electric field reaches high values in the vicinity of the electrode, causing the local ionization of air. Accelerated ions cause the glowing, that in specific conditions might be observed. Corona discharge is used for ionization (or deionization) in many industrial processes.

In the context of industrial safety, the most important parameter is the energy released during the electrostatic discharge, as it might be directly related to the minimum ignition energy (MIE) of a combustible substance.

\subsection{Typical industrial hazards}

In typical industrial conditions, spark discharge take place, if for some reason the grounding of the conductive body failed or was impossible to be realized. Only in such situation the charge will be accumulated on the body capacitance and released if specific conditions will be met. Usually, the literature gives a value of $3,2 \mathrm{kV} / \mathrm{mm}$ as a dielectric strength of the air. After the voltage of the conducting body will reach the given value, ESD will be observed, if grounded object will be placed close enough. It should be noted, that also the human body might be considered as a grounding object.

In general, electric charge will accumulate on the conductive body if either there is a physical separation of the material (or friction), like during the pneumatic transport of media or there is a strong electric field present, causing the object to electrify by the induction.

In some processes, the presence of dielectric bodies is inseparable. If transported medium is non-conductive, and is being poured, electric charge might accumulate. If there is a dielectric coating, or e.g. a foil is used, other types of electrostatic discharge might be observed: brush discharge, propagating brush discharge. In case of low radius of curvature of the electrode, corona discharge might be observed. These types of ESD, however, are not the scope of current study.

In general, to assess the actual energy $E$ released during the discharge, the initial $\left(V_{i}\right)$ and final $\left(V_{f}\right)$ voltage of the capacitor of the capacity $C$ should be measured. Then the energy released (from the capacitor) might be calculated from the formula:

$$
E=0,5 \cdot C \cdot\left(V_{i}^{2}-V_{f}^{2}\right)
$$

However, not whole energy stored within the circuit of the spark generator will be released during ESD [3-4]. In test conditions, the most reliable way is to determine both voltage $u$ and current $i$ over time and to integrate the results:

$$
E=\int i(t) u(t) d t
$$

\subsection{MIE of chosen substances}

In the industries, where gaseous hazardous atmospheres are present, minimum ignition energies are considerably lower than for dust atmospheres. The differences might reach as much as 5 orders of magnitude, or even more in some cases. ATEX directive provides the gas group classification, basing on minimum ignition current and other specific parameter, which is maximum experimental safe gap. The classification is crucial from the perspective of intrinsic safety of the equipment. In the table 1 chosen minimum ignition energies of the gas-air and dust-air mixtures were given. 
Table 1. Typical values of minimum ignition energies (MIE) of gases and dusts.

\begin{tabular}{|l|l|l|l|l|l|}
\hline Substance & MIE $[\mathbf{m J}]$ & Source & Substance & MIE [mJ] & Source \\
\hline Hydrogen & 0,011 & {$[6]$} & Pittsburg coal & 250 & {$[7]$} \\
\hline Butane & 0,26 & {$[7]$} & Zinc & 960 & {$[7]$} \\
\hline Methane & 0,28 & {$[6]$} & Wheat starch & 20 & {$[6]$} \\
\hline Acetylene & 0,017 & {$[6]$} & Sulphur & 15 & {$[6]$} \\
\hline
\end{tabular}

The energies necessary to ignite particular hazardous atmosphere might differ significantly. Among typical parameters that influence MIE value following should be noted: concentration, turbulence level, temperature, humidity (for dusts) and particle size distribution (for dusts). Therefore the following diagram might be useful to illustrate the hazards.

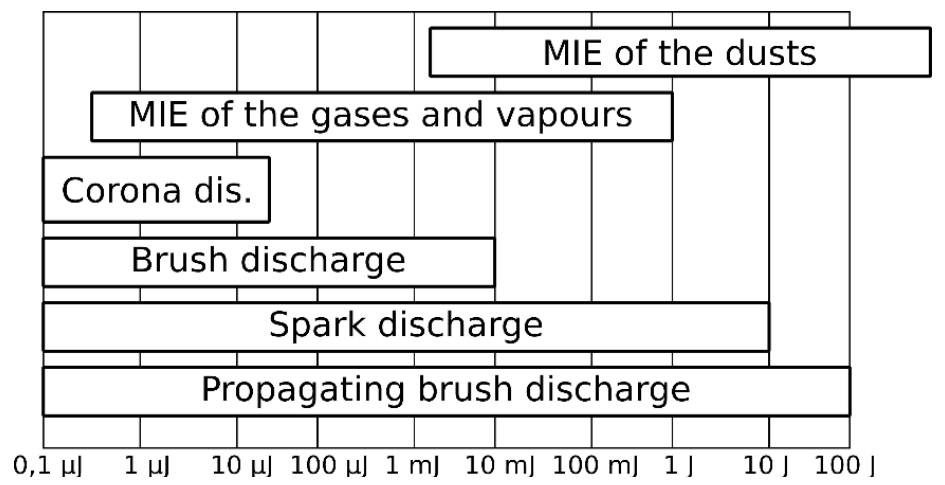

Fig. 1. Comparison of possible ranges of the energy released depending on a type of ESD and typical values of the minimum ignition energies of gases, vapors and dusts (https://www.ciop.pl/CIOPPortalWAR/file/56430/Materialy_informacyjne_nt_elstat_prezent.pdf, 15.08.2018).

\section{Research}

\subsection{Method}

In the current study, the spark generator was used to produce ESD for analyzing their course. Schematic layout of the discharge circuit and data acquisition system was shown in the Fig. 2. 


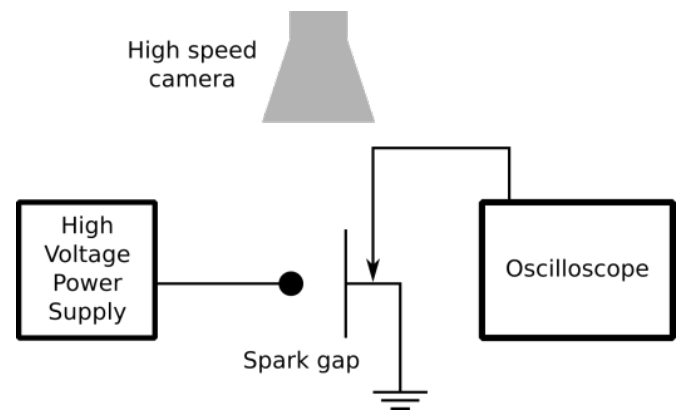

Fig. 2. Schematic layout of the discharge circuit and data acquisition system.

To generate high voltage, Glassman PL/LT series power supplies were used. The devices were capable of reaching a voltage of $150 \mathrm{kV}$ of both polarities. Power supply was connected to the spherical electrode of the diameter of $50 \mathrm{~mm}$. The other electrode was grounded metal plate of dimensions $160 \times 380 \mathrm{~mm}$.

It should be noted, that the energy of the discharge was not measured in present study. Therefore only high voltage probe was used to monitor the voltage waveforms. The oscilloscope used was Tektronix MSO 7000 compatible with the HV probe.

The data recorded was compared against the motion captured by the high speed camera. Phantom v1610 was used, allowing maximum frame rate of 1 Million fps at lowest resolution (128 x $16 \mathrm{px})$. The exposure time at maximum recording speed was equal to 452 ns, therefore additional lighting was introduced to ensure appropriate contrast.

The distance between the electrodes was changed to allow different values of the voltage necessary to start air breakdown and, in consequence, spark discharge. In presented study, the distance did not exceed $10 \mathrm{~mm}$.

\subsection{Course of spark discharge}

The nature of electrostatic spark discharge, the avalanche of the electrons, which equalize the charge of two conducting objects, causes the phenomenon to be rapid. Released energy is dependent of many factors. In laboratory conditions, the energy released is highly dependent on spark generator circuit parameters as the capacity, inductance and resistance. It is well described in the literature, that not all energy stored in the circuit capacitance is released in the form of spark discharge. Some of the energy is transferred into heat on the circuit resistance, usually constituting at least $10 \%$ of the whole energy stored in the circuit prior to the discharge [3] but may reach much higher values, reaching up to $60 \%$ in some conditions [4], typically in the circuits with high inductance.

Spark duration was also examined and described in the literature. In his study, Ono et al examined the influence of the humidity and spark duration on the minimum ignition energy of hydrogen-air mixture [8]. The parameter was approximately constant for spark durations ranging between $5 \mathrm{~ns}$ to $1 \mathrm{~ms}$.

In current study, the course of the electrostatic spark discharge was analyzed. Typical one was shown in the Fig. 3 for positive polarity of the spherical electrode and grounded planar electrode. The distance between the electrodes was approximately 8,6 mm. The discharge took place approximately at a voltage of $26 \mathrm{kV}$. 

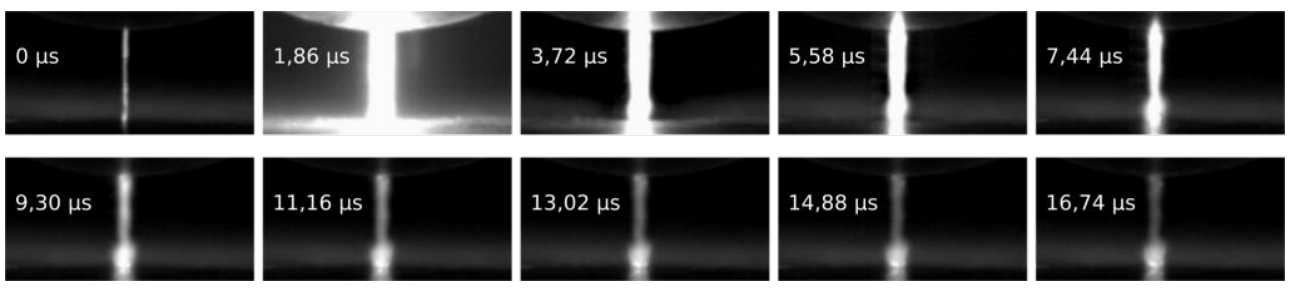

Fig. 3. Typical course of electrostatic spark discharge recorded for positive polarity of the spherical electrode at a distance of approx. $8,6 \mathrm{~mm}$ from grounded planar electrode.

As might be observed, most of the energy is usually released in the first part of the discharge, when after air gap breakdown the electron avalanche is observed. In the Fig. 3. a pre-discharge was also recorded, during which the gas was preliminary ionized. The second capture resembles the electron avalanche. It should be noted, that due to relatively high exposure time, most part of the actual discharge should be seen on the second capture (ended at 1,86 $\mu \mathrm{s}$ ). The following frames recorded the glowing of the ionized gas. It still allows the remaining charge to flow between the electrodes, but the observed glowing is connected mostly with the high temperature of the gas.

To fully understand the nature of the ESD, the voltage was recorded with the use of the oscilloscope. For above given discharge, following course of the discharge was observed.

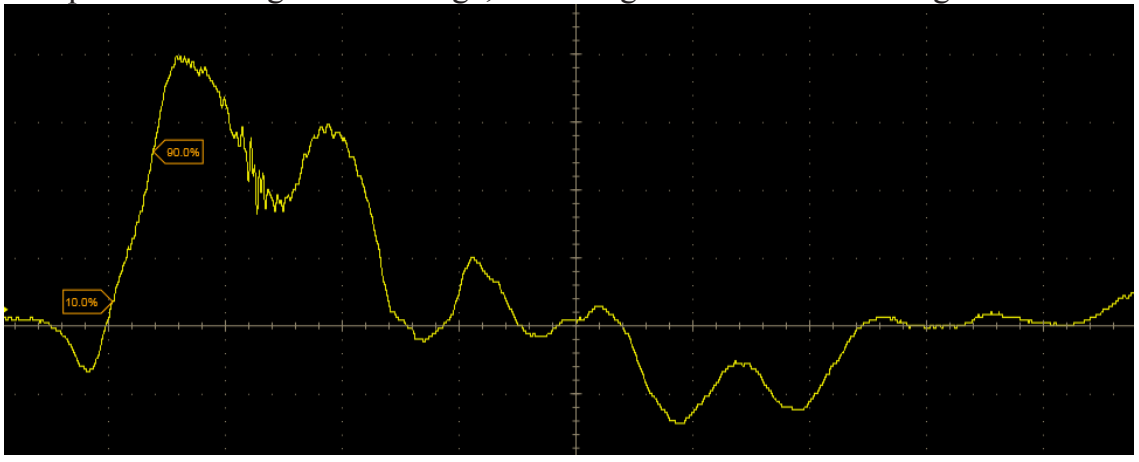

Fig. 4. Typical voltage waveform recorded during the electrostatic spark discharge (positive charge of the electrode, $40 \mathrm{~ns} / \mathrm{div})$.

Presented course of voltage changes shows the preliminary discharge started by the negative charge. After the partial air breakdown is observed, the discharge begins, releasing considerable energy. Two voltage peaks were observed, which is typical in specific conditions and was described in the literature, e.g. [4]. The first peak was observed approximately $40 \mathrm{~ns}$ after the initiation of the experiment, while the rising time was app. 20 ns. The most of the energy was released during first $100 \mathrm{~ns}$.

Next, the polarization of the electrode was changed to negative and the observed effects were shown in the Fig. 5.
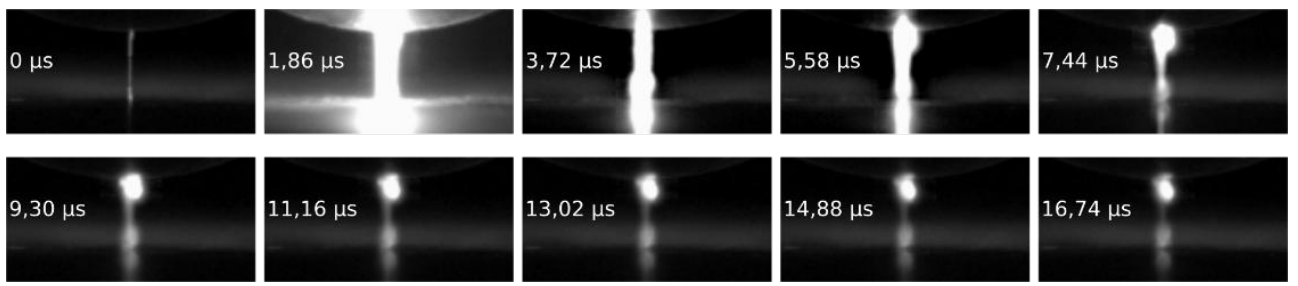
Fig. 5. Typical course of electrostatic spark discharge recorded for negative polarity of the spherical electrode at a distance of approx. $6,6 \mathrm{~mm}$ from grounded planar electrode.

In above mentioned test trial, the potential of the spherical electrode was raised up to $20 \mathrm{kV}$. The distance between the planar electrode and the spherical one was approximately $6,6 \mathrm{~mm}$. Similar phenomenon was observed: most of the energy was released during the first dozens of nanoseconds, what can be clearly seen on the oscillogram showed in the Fig. 6.

In case of negative polarity of the spherical electrode, the glowing of the ionized gas was considered to be more intense in the vicinity of the electrode. In previous case, the glowing was more durable close to the planar, grounded electrode. It is assumed, that it is caused by the weak ionization taking place for some time after the spark itself, present in the vicinity of the negative electrode. It should be understood, that the circuit of the spark generator was not completely discharged.

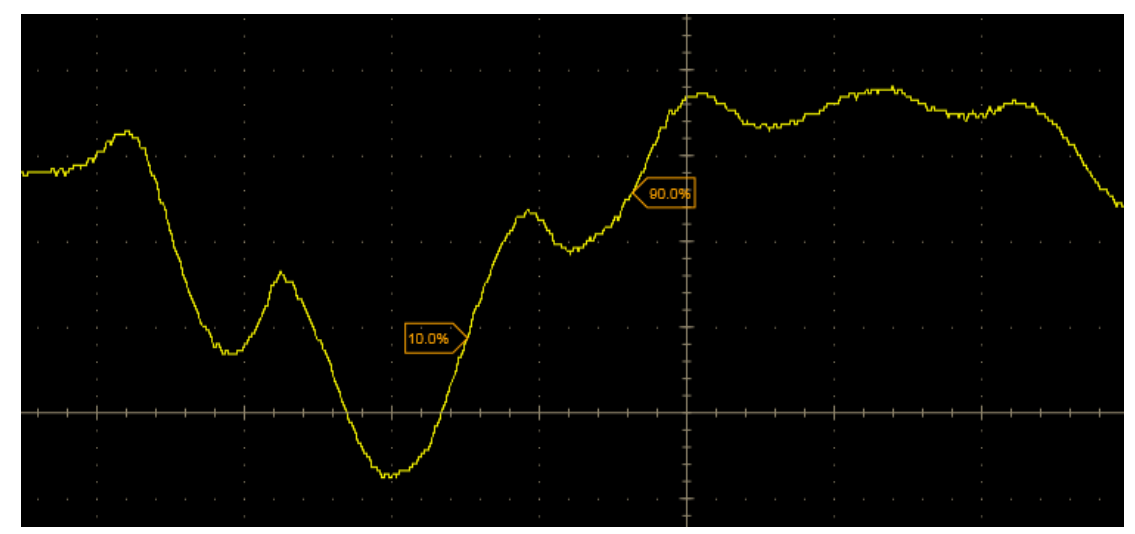

Fig. 6. Typical voltage waveform recorded during the electrostatic spark discharge (negative charge of the electrode, $40 \mathrm{~ns} / \mathrm{div}$ ).

Again two voltage peaks were observed, however, typically the first one was lower, than the second one.

To fully understand the nature of a spark discharge, it should be noted, that its power is considerably high, causing immediate ionization of the air, in which heat is being released to the vicinity of the spark gap. According to the classical ignition theory of Zeldovich, if the temperature rises above the lowest ignition temperature of a combustible mixture, the explosion (or a fire) will occur. In case of gaseous atmospheres, the energies necessary to cause the ignition are considerably lower, than is case of dust-air mixtures. It might be explained, that in case of gases, if the temperature threshold is achieved, homogenous combustion will take place. In case of solids, firstly the thermal decomposition of the material is observed, and afterwards, when the gaseous products are heated up to the threshold temperature, Zeldovich mechanism is observed. Therefore, in its principle, heat transfer mechanisms play crucial role and cause such considerable difference between the course of gaseous and dust atmospheres ignition.

In typical industrial conditions, spark discharge might be easily controlled. Appropriate grounding and electrical potential equalization of the conducing bodies, such as machinery housing, usually is a sufficient mean of protection. However the course of the discharge might be slightly different from the discharge observed in laboratory conditions (usually generated by the set of capacitors), the power of the discharge will be high enough to cause the ignition of the hazardous atmosphere. 


\section{Conclusions}

Basing on the literature and experimental study, the following conclusions might be made:

- In most of the industrial conditions, controlling of the electrostatic hazard would be necessary.

- In all technological processes, where combustible material will be used, the explosion hazard should be taken into consideration. Most of the combustible dusts are characterized by the minimum ignition energies significantly higher than the ones recorded for gases.

- Typical electrostatic spark discharge will last in the range of dozens of nanoseconds. The energy released will be usually high enough to ignite gaseous atmospheres in optimal conditions. The study showed, that the maximum exposure time is at least one order of magnitude higher than the ESD itself. It is possible to judge the occurrence (or not) of the ESD and to observed the glowing of the ionized air.

- The course of the ESD in variable conditions were analyzed within presented study. Both high speed camera and the oscilloscope were used. The outcomes showed, that the use of both of the devices simultaneously enable to describe both qualitatively and quantitatively the ESD.

- Electrostatic spark discharge should be considered as a significant hazard and the measures to limit the explosion risk should be taken. Presented method might be easily developed to estimate the energy released during the ESD and used in real conditions, for the sake of explosion safety estimation.

- $\quad$ The test method will be developed. The estimation of the energy released during the brush discharge, as well as the number of streamers created will be therefore possible.

The article presents results obtained within the National Program "Improvement of safety and working conditions", stage IV, task III.N.08 - supported by the Ministry of Science and Higher Education/National Centre for Research and Development, Poland (Central Institute for Labour Protection - National Research Institute is the program's main co-ordinator).

\section{References}

1. R.F. Eckhoff, Zeitschr. Physik. Chem. 231, 10 (2016)

2. Q. Liu, Y. Zhang, J. Appl. Phys. 116, 153302 (2014)

3. E. Randeberg, W. Olsen, R.F. Eckhoff, J. Electrost. 64 (2006)

4. S. Zhong, N. Miao, Q. Yu, W. Cao, J. Electrost. 73 (2015)

5. Directive 2014/34/EU of the European Parliament and of the Council of 26 February 2014 on the harmonisation of the laws of the Member States relating to equipment and protective systems intended for use in potentially explosive atmospheres.

6. H. Haase, Electrostatic Hazards, Their Evaluation and Control (Verlag Chemie, Weinheim, 1977)

7. V. Babrauskak, Ignition Handbook (Fire Science Publishers, Issaquah WA, 2003)

8. R. Ono, M. Nifuku, S. Fujiwara, S. Horiguchi, T Oda, J. Electrost. 65 (2007) 